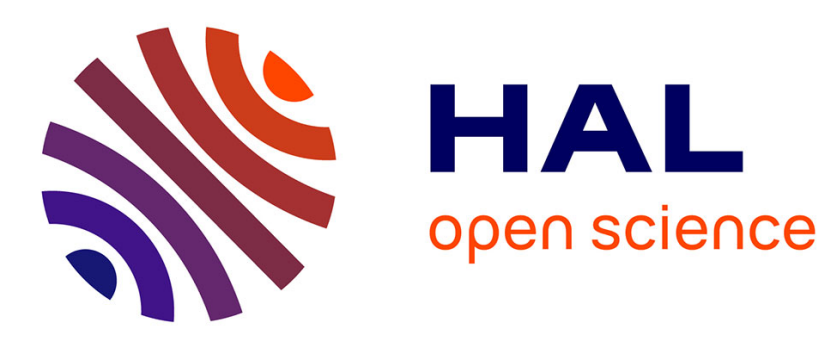

\title{
Characterization of an optimized off-diagonal GMI-based magnetometer
}

\author{
Basile Dufay, Sébastien Saez, Christophe Dolabdjian, Arthur Yelon, David \\ Ménard
}

\section{- To cite this version:}

Basile Dufay, Sébastien Saez, Christophe Dolabdjian, Arthur Yelon, David Ménard. Characterization of an optimized off-diagonal GMI-based magnetometer. IEEE Sensors Journal, 2013, 13 (1), pp.379 388. 10.1109/JSEN.2012.2216521 . hal-00824131

\section{HAL Id: hal-00824131 \\ https://hal.science/hal-00824131}

Submitted on 21 May 2013

HAL is a multi-disciplinary open access archive for the deposit and dissemination of scientific research documents, whether they are published or not. The documents may come from teaching and research institutions in France or abroad, or from public or private research centers.
L'archive ouverte pluridisciplinaire HAL, est destinée au dépôt et à la diffusion de documents scientifiques de niveau recherche, publiés ou non, émanant des établissements d'enseignement et de recherche français ou étrangers, des laboratoires publics ou privés. 


\title{
Characterization of an optimized off-diagonal GMI-based magnetometer
}

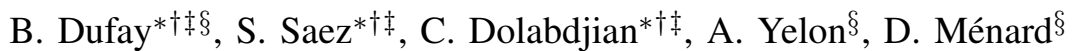 \\ *Université de Caen Basse-Normandie, UMR 6072 GREYC, F-14032 Caen, France \\ ${ }^{\dagger}$ ENSICAEN, UMR 6072 GREYC, F-14032 Caen, France \\ ${ }^{\ddagger}$ CNRS, UMR 6072 GREYC, F-14032 Caen, France \\ $\S$ École Polytechnique de Montréal, département de génie physique \& Regroupement québécois des matériaux de \\ pointe, Montréal, Québec, Canada H3C3A7
}

\begin{abstract}
An optimized giant magneto-impedance effect magnetometer has been developed, based upon an overall analysis of the measurement chain, including physical material properties, associated detection coil parameters, and equivalent magnetic noise performances. The field response model for the sensing element and the noise model yield good agreement with experimental results. The noise performance of the magnetometer, approximately $1.7 \mathrm{pT} / \sqrt{\mathrm{Hz}}$ in the white noise region, with a band-pass of about $70 \mathrm{kHz}$, is competitive with that of other technologies. Present limitations are clearly established, leaving room for further improvements.
\end{abstract}

Index Terms-Giant magneto-impedance (GMI), magnetometer, noise.

\section{INTRODUCTION}

The giant magneto-impedance (GMI) effect arises from the change of the ac permeability of a magnetic conductor submitted to a change of external magnetic field. It has attracted considerable attention over the past two decades, mainly because of its potential for high sensitivity magnetometry [1].

An important characteristic of performance in high sensitivity magnetometry is the equivalent magnetic noise, since it limits the utlimate sensing performance. We have previously reported that the limit of GMI magnetometers is currently determined by electronic conditioning noise, rather than by the intrinsic noise of the GMI sensing element [2], [3], [4]. Consequently, it will be advantageous to increase the voltage sensitivity, $S_{v}$, until the electronic conditioning noise and the sensor noise are comparable. The voltage sensitivity is defined as the voltage variation corresponding to the magnetic field variation and is expressed in $\mathrm{V} / \mathrm{T}$. It is related to the intrinsic sensitivity, $S_{\Omega}$, expressed in $\Omega / \mathrm{T}$, and to the optimal excitation current amplitude as discussed in [5], [6].

In ref. [6], we reported an investigation of the choice of GMI material, and of material optimization with respect to the voltage sensitivity. Another approach to improving sensitivity is the choice of a two port network configuration, in which the GMI element is associated with a pick-up coil (this is sometimes referred to as off-diagonal GMI [7] or orthogonal fluxgate in the fundamental mode [8]). In a recent paper [9], we discussed the effect of such a choice on noise performance. Based upon these investigations, we present here a fully optimized magnetometer, operating in a field-locked loop.
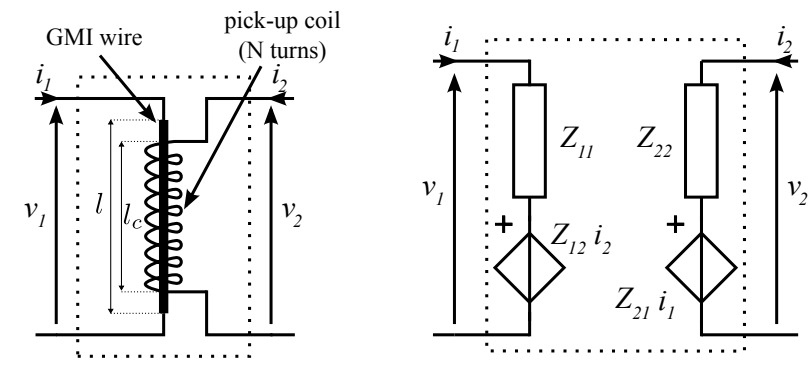

Figure 1: Sensing element schematic and its associated twoport network model. It illustrates the different terms of the impedance matrix given in (1).

The paper is organized as follows. Section II recalls those aspects of GMI needed to predict the field response of the complete sensing element. Section III is dedicated to the description of the electronic conditioning circuitry and the associated noise model. This, in combination with the physical model of GMI, allows us to predict noise performance and its dependance upon the parameters of the sensing element or of the measurement chain. Section IV presents the resulting optimized magnetometer, and its performance. Finally, a general conclusion is given in section $\mathrm{V}$.

\section{PRINCIPLE OF THE SENSING ELEMENT}

\section{A. Basic definitions}

The sensing element is made of a thin pick-up coil wound directly on a $100 \mu \mathrm{m}$ diameter $\mathrm{CoFeSiB}$ amorphous ferromagnetic wire. Details concerning the GMI wire are presented in [6] (sample designation c3 with saturation magnetization $M_{s}=561 \mathrm{kA} / \mathrm{m}$, phenomenological Gilbert damping parameter $\alpha=0.020$ and electrical resistivity $\rho=129 \mu \Omega . \mathrm{cm}$ ). This sample was chosen because of the ease of its use in the GMIcoil structure. The length of the pick-up coil, $l_{c}$, was equal to that of the wire, $l$, and is around $2.5 \mathrm{~cm}$. The number of turns of the coil, $N$, can be adjusted by connecting one, two or three coil layers in series, with approximately 500 turns/layer. A schematic of the sensing element is presented in Fig. 1 along with the associated two-port network model.

This sensing element could be described by its field- 
dependent impedance matrix $[Z(B)]$, where $B\left(=\mu_{0} H\right)^{\mathrm{i}}$ is the external magnetic induction, as

$\left(\begin{array}{l}v_{1} \\ v_{2}\end{array}\right)=[Z(B)]\left(\begin{array}{c}i_{1} \\ i_{2}\end{array}\right)=\left[\begin{array}{ll}Z_{11}(B) & Z_{12}(B) \\ Z_{21}(B) & Z_{22}(B)\end{array}\right]\left(\begin{array}{l}i_{1} \\ i_{2}\end{array}\right)$.

For operation in a field closed loop, the external magnetic field may be re-written as

$$
B=B_{0}+b(t)
$$

where $B_{0}$ is a static working point and $b(t)$ is the small signal variation of the magnetic field around $B_{0}[10]$. Using a first order Taylor expansion, each term of the impedance matrix thus becomes

$$
Z_{i j}(B)=Z_{i j_{0}}+\left.\frac{\partial Z_{i j}(B)}{\partial B}\right|_{B=B_{0}} . b(t)
$$

where $Z_{i j_{0}}=Z_{i j}\left(B_{0}\right)$ and $\partial Z_{i j}(B) / \partial B$ is the intrinsic sensitivity of each term of the impedance matrix, in units of $\Omega / \mathrm{T}$, and $b(t)$ is the sensed magnetic field.

As suggested by Eq. (1), there are four different configurations of excitation and detection, referring to the four elements of the impedance matrix. In [9], we concluded that the offdiagonal configuration, corresponding to an excitation current flowing through the GMI wire, with voltage detection at the pick-up coil terminals, is the most promising for improving noise performance for excitation frequency limited to a few $\mathrm{MHz}$. Consequently, the sensing element is operated in this configuration in what follows.

\section{B. Modeling the field response of the sensing element}

Classical modeling of the GMI effect involves the surface impedance tensor $\left[\zeta_{s}\right]$ which links the electric, $e$, and magnetic, $h$, field components at the wire surface [11], as

$$
\left(\begin{array}{c}
e_{\phi} \\
e_{z}
\end{array}\right)=\left[\zeta_{s}\right]\left(\begin{array}{c}
h_{\phi} \\
h_{z}
\end{array}\right)=\left[\begin{array}{ll}
\zeta_{\phi z} & \zeta_{\phi \phi} \\
\zeta_{z z} & \zeta_{z \phi}
\end{array}\right]\left(\begin{array}{c}
h_{\phi} \\
h_{z}
\end{array}\right)
$$

where circumferential and axial components are designated by indices $\phi$ and $z$, respectively.

The calculation of $\left[\zeta_{s}\right]$ is based on two steps: (1) the determination of the static equilibrium position of the magnetization, and (2) the simultaneous solution of Maxwell's equations and the Landau-Lifshitz equation of motion for the magnetization.

First, the static equilibrium position is obtained by determining the angle, $\theta_{M}$, of the magnetization direction which minimizes the free energy density of the system, $U_{0}$. The latter, including terms of Zeeman energy and of an effective anisotropy field, is given by [12]

$$
\frac{U_{0}}{\mu_{0} M_{s}}=-H_{z} \cos \theta_{M}-H_{\varphi} \sin \theta_{M}-\frac{1}{2} H_{k} \cos ^{2}\left(\theta_{k}-\theta_{M}\right)
$$

where $M_{s}$ is the saturation magnetization amplitude, $H_{z}$ is the axial applied magnetic field, $H_{\varphi}$ is the circumferential static magnetic field induced by the static bias current $I_{d c}=$ $2 \pi a H_{\varphi}$ ( $a$ is wire radius), and $H_{k}$ and $\theta_{k}$ are the magnitude

\footnotetext{
${ }^{\mathrm{i}} \mathrm{Here}, B$ does not include the magnetization of the wire.
}

and direction of the anisotropy field. Angles $\theta_{M}$ and $\theta_{k}$ are defined from the micro-wire axis direction.

As in ref. [12], we consider a new coordinate system, $\left(\overrightarrow{n_{r}}, \overrightarrow{n_{\perp}}, \overrightarrow{n_{\|}}\right)$, based on cylindrical coordinates with $\overrightarrow{n_{\|}}$aligned with the direction of the magnetization vector. The simultaneous solution of Maxwell's equations and the LandauLifshitz equation in this system leads to the separation of the surface tensor into a magnetic, $Z_{M}$, and non-magnetic, $Z_{N}$, component. It yields

$$
\left(\begin{array}{c}
e_{\perp} \\
e_{\|}
\end{array}\right)=\left(\begin{array}{cc}
0 & -Z_{N} \\
Z_{M} & 0
\end{array}\right)\left(\begin{array}{c}
h_{\perp} \\
h_{\|}
\end{array}\right)
$$

which is related to $\zeta_{s}$ through a coordinate rotation by an angle $\theta_{M}$, so that

$$
\left\{\begin{array}{ccc}
\zeta_{z z} & = & Z_{M} \cos ^{2} \theta_{M}+Z_{N} \sin ^{2} \theta_{M} \\
\zeta_{\varphi \varphi} & = & -Z_{M} \sin ^{2} \theta_{M}-Z_{N} \cos ^{2} \theta_{M} \\
\zeta_{\varphi z} & = & -\zeta_{z \varphi}=\left(Z_{M}-Z_{N}\right) \sin \theta_{M} \cos \theta_{M}
\end{array} .\right.
$$

Then, $Z_{N}$ and $Z_{M}$ are given by

$$
Z_{N}=k_{N} \rho \frac{J_{0}\left(k_{N} a\right)}{J_{1}\left(k_{N} a\right)}, Z_{M}=k_{M} \rho \frac{J_{0}\left(k_{M} a\right)}{J_{1}\left(k_{M} a\right)}
$$

where $J_{0}$ and $J_{1}$ are first kind Bessel functions, $k_{M}$ and $k_{N}$ are the magnetic and non-magnetic components of the propagation vectors of the EM wave with $k_{M}=k_{N} \sqrt{\frac{\mu_{M}}{\mu_{0}}}$ and $k_{N}=\frac{1-\jmath}{\delta_{N}}$. Here, $\delta_{N}\left(\delta_{N}=\sqrt{\frac{2 \rho}{\omega_{p} \mu_{0}}}\right)$ represents the non-magnetic skin depth; $\rho$ is the resistivity of the material, $\mu_{0}$ is the permeability of vacuum and $\omega_{p}\left(\omega_{p}=2 \pi f_{p}\right)$ is the working frequency. The exact expression for apparent magnetic permeability $\mu_{M}$ can be found in [2].

Finally, the electric and magnetic fields at the wire surface can be related to current and voltage [13], as:

$$
\begin{aligned}
v_{1} & =l e_{z}, \\
i_{1} & =2 \pi a h_{\varphi}, \\
v_{2} & =-2 \pi a N e_{\varphi}, \\
i_{2} & =\frac{l_{c}}{N} h_{z} .
\end{aligned}
$$

Combining Eqs. (1), (4), (7) and (9a) to (9d), we can predict the magnetic response of the whole impedance matrix.

Here, we must point out that some rough approximations have been made in this model. Indeed, the possible free space between the GMI wire outer diameter and coil inner diameter is not taken into account, nor is the propagation of the EM wave into the medium surrounding the GMI wire, nor, especially, the resistivity of the coil.

At this point, the parasitic capacitance of the coil must be considered. It may be due to turn-to-turn capacitance or turnto-core capacitance. The simplest and most classical way of taking it into account is to consider a capacitance in parallel with the inductance, as shown in Fig. 2. In such a case, the new expression, $\left[Z^{\prime}\right]$, for the impedance matrix is

$$
\left(\begin{array}{cc}
Z_{11}^{\prime} & Z_{12}^{\prime} \\
Z_{21}^{\prime} & Z_{22}^{\prime}
\end{array}\right)=\left(\begin{array}{cc}
Z_{11}-\frac{\jmath Z_{12} Z_{21} C_{p} \omega_{p}}{1+\jmath Z_{22} C_{p} \omega_{p}} & \frac{Z_{12}}{1+\jmath Z_{22} C_{p} \omega_{p}} \\
\frac{Z_{21}}{1+\jmath Z_{22} C_{p} \omega_{p}} & \frac{Z_{22}}{1+\jmath Z_{22} C_{p} \omega_{p}}
\end{array}\right)
$$




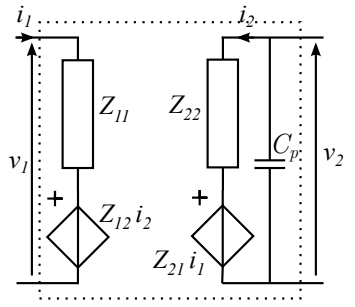

(a)

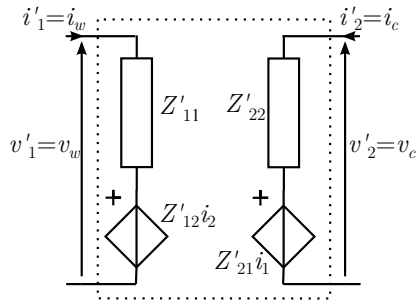

(b)
Figure 2: Modification of the GMI-coil structure by the stray capacitance, $C_{p}$, (a), with its two port equivalent model, $\left[Z^{\prime}\right]$, (b). In what follows, the new two-port model designation, $\left[Z^{\prime}\right]$, will be replaced by the old one, $[Z]$, in order to simplify the notation. The coil stray capacitance is then implicitly considered.

where $Z_{11}=\frac{l}{2 \pi a}\left(Z_{M} \cos ^{2} \theta_{M}+Z_{N} \sin ^{2} \theta_{M}\right)$, $Z_{12}=Z_{21}=N\left(Z_{N}-Z_{M}\right) \sin \theta_{M} \cos \theta_{M} \quad$ and $Z_{22}=\frac{2 \pi a N^{2}}{l_{c}}\left(Z_{M} \sin ^{2} \theta_{M}+Z_{N} \cos ^{2} \theta_{M}\right)$. In what follows, the two-port model designation, $\left[Z^{\prime}\right]$, will be replaced by $[Z]$, in order not to complicate the notation. The stray capacitance of the coil is then implicitly considered.

\section{Optimization}

The sensing element is used in the off-diagonal configuration. That is, the excitation current flows through the GMI wire. In this case, we may assume that the optimal excitation current amplitude, discussed in [6], is related only to wire properties, and does not depend upon coil parameters. Assuming that the GMI sample is operated using its optimal excitation current amplitude, we want to increase the intrinsic sensitivity of the off-diagonal term, $\partial Z_{21} / \partial B$. At first sight, according to Eq. 10, it seems possible to increase $Z_{21}$ by increasing the number of coil turns, $N$, so that its sensitivity will increase, in scale.

Keeping that goal in mind, Fig. 3 shows the maximum intrinsic sensitivity as a function of excitation frequency, $f_{p}$, for several values of $N$ and compares it with model predictions. At low frequency, the presence of $C_{p}$ is not noticeable since the two models, with or without parasitic capacitance, are similar. As frequency increases, $C_{p}$ induces resonant behavior, so that the model which includes it predicts higher sensitivity than the one without. At high frequency, it predicts a decrease, in agreement with experiment. Clearly, this occurs at lower $f_{p}$ for higher $N$, leading to the observation of an optimal operating frequency, which depends upon $N$.

This optimal operating frequency may not be the frequency at which the GMI effect, and thus the sensitivity, are maximum, with respect to physical properties of the wire [6]. As GMI effect usually increases with excitation current frequency, up to a certain point, a lower capacitance value is clearly preferable (Fig. 3). Moreover, the sensitivity scales roughly with the number of turns, so that a value of $N$ as high as possible is preferable. Nevertheless, according to Fig. 4, if the parasitic capacitance is around $15 \mathrm{pF}$, the increase of $N$, going from 1200 to 1800 turns, has no effect on the sensitivity. If

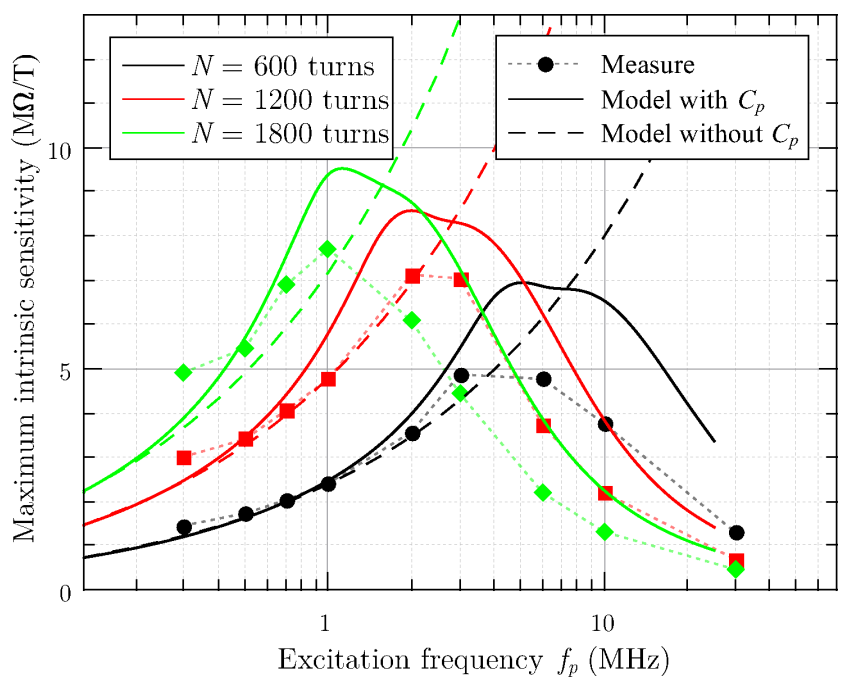

Figure 3: Maximum intrinsic sensitivity, $\partial Z_{21} / \partial B$, as a function of excitation frequency, $f_{p}$, for several values of $N$. The number of turns was adjusted by connecting coil layers in series. The DC bias current, $I_{d c}$, was set to $6 \mathrm{~mA}$, the optimal value, according to [6]. Symbols illustrates experimental values whereas lines are obtained by simulation with $H_{k}=40 \mathrm{~A} / \mathrm{m}, \theta_{k}=85^{\circ}$ and assuming a parasitic capacitance of $10 \mathrm{pF}$. Dashed lines show theoretical results, when the parasitic capacitance is not considered.

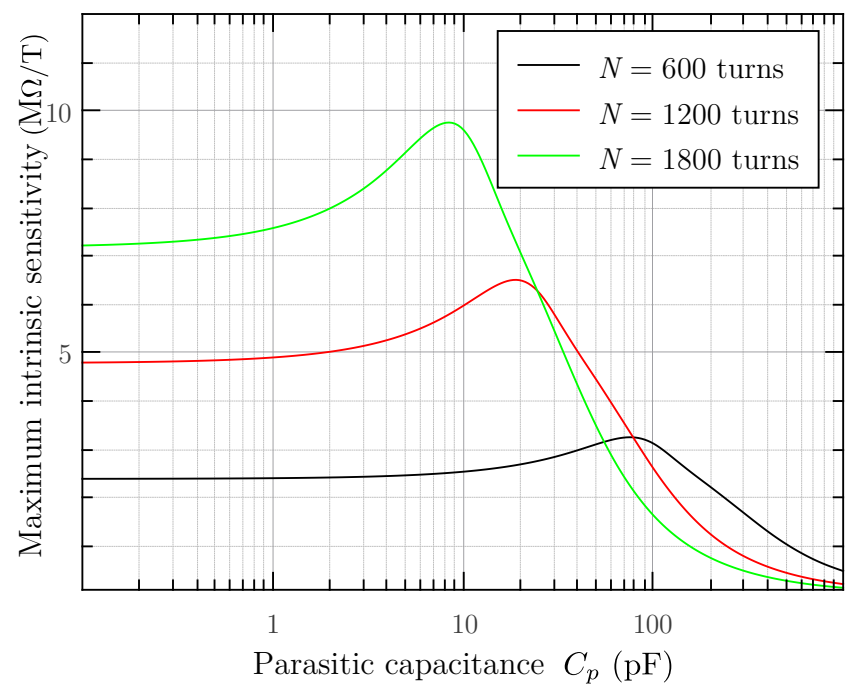

Figure 4: Maximum intrinsic sensitivity, $\partial Z_{21} / \partial B$, as a function of $C_{p}$. Each curve represents a different number, $N$, of turns of the coil. The simulation settings are the same as in Fig. 3, with an excitation frequency, $f_{p}$, of $1 \mathrm{MHz}$.

$C_{p}$ is as low as possible, increasing $N$ increases the sensitivity for as wide a range of values of $N$ as possible. The parameter, $C_{p}$, appears to determine the limit of the performance of the off-diagonal structure.

Finally, the number of turns is chosen to be $N \approx 1000$, well adapted for working at an excitation frequency around $1 \mathrm{MHz}$. The coil is made of two layers, of which the first is wound directly on the total length of the amorphous wire (without any gap between the wire and the first layer, except for the 


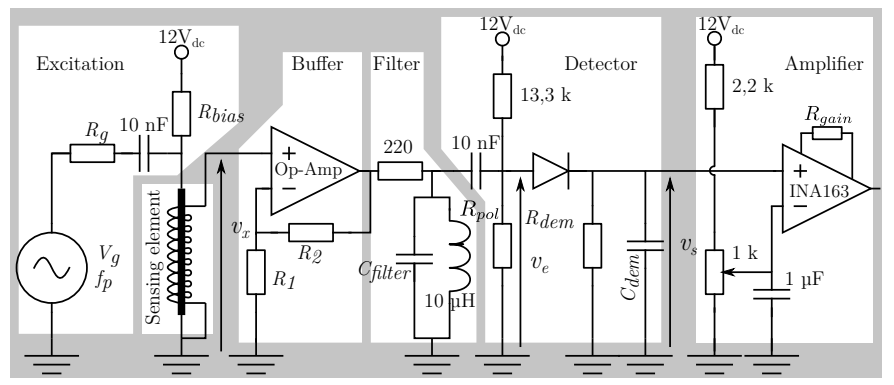

Figure 5: Schematic of the optimized open-loop measuring chain. If not indicated otherwise, component values are: $R_{g}=750 \Omega, R_{\text {bias }}=2,2 \mathrm{k} \Omega, R_{1}=100 \Omega, R_{2}=500 \Omega$, $C_{\text {filter }}=2,2 \mathrm{nF}, R_{\text {pol }}=1 \mathrm{k} \Omega, R_{\text {dem }}=10 \mathrm{k} \Omega, C_{\text {dem }}=1 \mathrm{nF}$ and $R_{\text {gain }}=30 \Omega\left(G_{I N A}=200\right)$.

isolation of the coil wire, around $5 \mu \mathrm{m}$ ). The sensing element is $2.5 \mathrm{~cm}$ long.

\section{NOISE MODELING}

\section{A. Electronic conditioning}

The open-loop measurement chain, presented in Fig 5, consists of several stages. First, the excitation stage is based on a sine voltage generator, converted to a current source by the injection resistor $R_{g}$, as described in [9]. The value of $R_{g}$ is adjusted so that the current amplitude is at least $10 \mathrm{~mA}$, as determined in [6], limited principally by electronic conditioning. In addition, a static bias current of $6 \mathrm{~mA}_{\mathrm{dc}}$ is applied to the GMI wire through the resistor $R_{\text {bias }}$. As discussed in Sec. II-C, the excitation frequency is set near $1 \mathrm{MHz}$, tuned to the resonance of the sensing coil.

The next stage is the sensing element, described in Sec. II. It is followed by a voltage buffer, consisting of an OP37 operational amplifier with a non-inverting gain of 6 , determined by resistors $R_{1}$ and $R_{2}$. It provides a high input impedance for measurement of the voltage across the coil terminals. However, it places limits upon the dynamics of the output signal of the sensing element, due to slew-rate and saturation.

The fourth stage is an RLC filter, inserted at the buffer output, tuned to the excitation frequency. Its purpose is to reduce noise, due to spurious harmonics created in the buffer, or to non-linear behavior of the sensing element.

Following this, the detection stage is based on a diode peak detector, whose utility, in rejecting phase noise has been demonstrated in ref. [3]. The peak detector consists of a high speed diode, statically polarized by the resistor $R_{p o l}$ above the detection threshold. It is followed by an RC filter to ground. In ref. [10], the use of a peak detector is particularly appropriate, used in combination with a pulsed current excitation stage (based on a relaxation oscillator), leading to a decrease in the resulting amplitude noise. Here we do not use pulse current excitation, since it does not permit fine tuning of the excitation current amplitude. This is an important operating parameter, as discussed in ref. [6]. Finally, the output signal is fed to an instrumentation amplifier, INA163, whose gain, $G_{I N A}$, is set by the resistor, $R_{\text {gain }}$.

\section{B. Noise model}

Based on the noise model presented in ref. [9], we are able to evaluate the noise performance of the system, and to determine its optimal use. First, we investigate those noise sources which contribute to the high frequency white noise level near the carrier frequency. Then, we analyze noise sources appearing at low frequency, after demodulation.

\section{1) Noise sources appearing at high frequency:}

We focus here on the white noise in the range of 1 to $10 \mathrm{kHz}$ above and below the carrier frequency, $f_{p}$, which will be shifted to low frequency after demodulation. We examine the noise sources ${ }^{\mathrm{ii}}$ at each stage.

a) The generator:

$e_{n_{1}} \quad$ The noise spectral density associated with the generator delivering the excitation signal; it includes both phase and amplitude noise, $\alpha_{n}$ and $\theta_{n}$.

$e_{n_{R g}} \quad$ The noise spectral density associated with thermal fluctuations of resistors $R_{g}$ and $R_{\text {bias }}$ in parallel.

b) The sensing element:

$e_{n_{Q}} \quad$ The noise spectral density associated with thermal fluctuations of the sensing element.

c) The voltage buffer:

$e_{n_{b u f f}} \quad$ The voltage noise spectral density of the $e_{n}-$ $i_{n}$ model of the operational amplifier used in the buffer stage.

$i_{n_{b u f f}} \quad$ The current noise spectral density of the $e_{n}-i_{n}$ model of the operational amplifier used in the buffer stage.

$e_{n_{R_{1}}}, e_{n_{R_{2}}}$ The noise spectral densities associated with thermal fluctuations of resistors $R_{1}$ and $R_{2}$ respectively.

\section{d) The filter:}

$e_{n_{R_{f}}} \quad$ The noise spectral density associated with thermal fluctuations of the $220 \Omega$ resistor of the band-pass filter.

\section{e) The peak detector:}

$e_{n_{R_{p o l-H F}}}$ The noise spectral density associated with thermal fluctuations of the $1 \mathrm{k} \Omega$ resistor used in the static polarization of the diode.

Signal instabilities of sinusoidal sources are generally characterized by the single-sideband noise spectral density (NSD) expressed in decibels below the carrier per hertz $(\mathrm{dBc} / \mathrm{Hz})$. Assuming that noise sources are uncorrelated, the noise spectral density from the excitation stage, $e_{n_{g}}$, is given by

$$
\begin{aligned}
e_{n_{g}}^{2} & =e_{n 1}^{2}+e_{n R_{g}}^{2} \\
& =\left(\frac{V_{g} / \sqrt{2}}{10^{-148 / 20}}\right)^{2}+4 k_{B} T\left(R_{g} \| R_{\text {bias }}\right)
\end{aligned}
$$

where $V_{g}$ is the generator voltage output, assuming a dynamic range of $148 \mathrm{dBc} / \mathrm{Hz}$ [14].

\footnotetext{
${ }^{i i}$ Noise sources are expressed in noise spectral density in $\mathrm{V}^{2} / \mathrm{Hz}$.
} 
The noise, $e_{n_{Q}}$, from the sensing element is due to thermal fluctuations of the two-port network. We may distinguish two Johnson noise sources

$$
\begin{aligned}
e_{n_{G M I}}^{2}(f) & =4 k_{B} T \Re\left(Z_{11_{0}}\left(\omega_{p}\right)\right), \\
e_{n_{\text {coil }}}^{2}(f) & =4 k_{B} T \Re\left(Z_{22_{0}}\left(\omega_{p}\right)\right),
\end{aligned}
$$

which represent the active power fluctuations of the GMI element and of the coil. Those noise sources may be expressed at the sensor output, in the off-diagonal configuration, as

$$
e_{n_{Q}}^{2} \approx\left(\frac{\left|Z_{21_{0}}\left(\omega_{p}\right)\right|}{\left|Z_{11_{0}}\left(\omega_{p}\right)\right|+R_{g}}\right)^{2} e_{n_{G M I}}^{2}+e_{n_{c o i l}}^{2} .
$$

The voltage noise of the operational amplifier, $e_{n_{\text {buff }}}(=3 \mathrm{nV} / \sqrt{\mathrm{Hz}}$ [15]), and the noise arising from $R_{1}$ are seen as voltage noise at the buffer stage input. The noise from $R_{2}$ is seen at the buffer output. The current noise, $i_{n_{b u f f}}(=0.8 \mathrm{pA} / \sqrt{\mathrm{Hz}}[15])$, acts as a voltage noise at the buffer input, through the static impedance of the coil and the resistor $R_{1}$. Considering a buffer stage gain, $G_{b u f f e r}=1+R_{2} / R_{1}$, and an attenuation from the band-pass filter, $G_{\text {filter }}$, the noise at the demodulator input is given by

$$
\begin{aligned}
e_{n f_{1}}^{2}= & G_{\text {filter }}^{2}\left\{4 k_{B} T R_{2}+G_{b u f f e r}^{2}\left[e_{n_{b u f f}}^{2}\right.\right. \\
& +4 k_{B} T R_{1}+i_{n_{b u f f}}^{2}\left(\left(Z_{22_{0}}\left(\omega_{p}\right)\right.\right. \\
& \left.\left.\left.\left.-\frac{Z_{12_{0}}\left(\omega_{p}\right) Z_{21_{0}}\left(\omega_{p}\right)}{Z_{11_{0}}\left(\omega_{p}\right)+R_{g}}\right)^{2}+R_{1}^{2}\right)\right]\right\} .
\end{aligned}
$$

Then, the noise spectral density, $e_{n_{R_{f}}}$, due to the band-pass filter resistor, is seen as a noise source at the filter input. In contrast, $e_{n_{R p o l-H F}}$, appears at the demodulation stage input. These two sources contribute to the wide band noise at the demodulation input as

$$
\begin{aligned}
e_{n f_{2}}^{2} & =e_{n_{R p o l-H F}}^{2}+G_{f i l t e r}^{2} e_{n_{R f}}^{2} \\
& =4 k_{B} T R_{\text {pol }}+G_{\text {filter }}^{2} 4 k_{B} T R_{f} .
\end{aligned}
$$

Finally, assuming that all noise sources are uncorrelated, the total wide-band noise spectral density, $e_{n W B}$, at the demodulator input, is given by

$$
\begin{aligned}
e_{n W B}^{2}= & G_{b u f f e r}^{2} G_{f i l t e r}^{2}\left[\left(\frac{Z_{21_{0}}\left(\omega_{p}\right)}{Z_{11_{0}}\left(\omega_{p}\right)+R_{g}}\right)^{2} e_{n g}^{2}+e_{n Q}^{2}\right] \\
& +e_{n f}^{2}
\end{aligned}
$$

where $e_{n_{f}}^{2}=e_{n f_{1}}^{2}+e_{n f_{2}}^{2}$.

2) Noise sources appearing at low frequency after demodulation:

Several low frequency noise sources appear after demodulation. We can distinguish:

\section{a) The detector:}

$e_{n_{R p o l-B F}}$ The low frequency noise spectral density of the resistor, $R_{\text {pol }}$, (used for the diode static polarization).

$e_{n_{\text {Rdem }}}$ The noise spectral density of the resistor, $R_{d e m}$, placed at the demodulator output.

\section{b) The instrumentation amplifier:}

$e_{n_{A m p l}}$

The voltage noise spectral density of the instrumentation amplifier, INA163, whose evaluation involves four distinct noise sources. These are (I.) the voltage, $e_{n_{I N A}}(=1 \mathrm{nV} / \sqrt{\mathrm{Hz}}$ [16]), and (II.) the current, $i_{n_{I N A}}(=0.8 \mathrm{pA} / \sqrt{\mathrm{Hz}}[16])$, noise spectral densities of the $e_{n}-i_{n}$ model of the amplifier, (III.) the noise from the resistor, $R_{\text {gain }}$, which sets the gain and (IV.) the noise from the output stage, $e_{n_{I N A-S}}(=60 \mathrm{nV} / \sqrt{\mathrm{Hz}}$ [16]). Given an amplifier gain, $G_{I N A}$, we obtain

$$
\begin{aligned}
e_{n_{A m p l}}^{2}= & 4 k_{B} T R_{\text {gain }}+e_{n_{I N A}}^{2}+\frac{e_{n_{I N A-S}}^{2}}{G_{I N A}^{2}} \\
& +i_{n_{I N A}}^{2}\left(R_{d e m}^{2}+1000^{2}\right)
\end{aligned}
$$

We also have

$$
e_{n_{\text {Rdem }}}^{2}=4 k_{B} T R_{d e m}
$$

and

$$
e_{n_{R p o l-B F}}^{2}=4 k_{B} T R_{p o l}
$$

since low frequency fluctuations are transmitted with no modification through the diode.

Finally, the low frequency noise spectral density, $e_{n L F}$, appearing after demodulation is given by

$$
e_{n L F}^{2}=e_{n_{R d e m}}^{2}+e_{n_{R p o l-B F}}^{2}+e_{n_{A m p l}}^{2} .
$$

\section{3) Magnetic noise sources:}

The small signal magnetic field to be measured, $b(t)$, appearing in Eqs. (2) and (3), is the sum of the useful signal and noise from magnetic sources, $b_{\text {noise }}$, which depend upon the three different kind of uncorrelated magnetic noise sources, so that

$$
b_{\text {noise }}^{2}=b_{\text {ext }}^{2}+b_{G M I}^{2}+g_{c}^{2} i_{\text {det }}^{2}
$$

where $b_{G M I}$ represents the intrinsic magnetic noise of the GMI, arising from the thermal fluctuations of the magnetization direction, $b_{\text {ext }}$ is the ambient magnetic noise and $g_{c} i_{d e t}$ is the noise due to low frequency current noise, $i_{\text {det }}$, of the conditioning electronics, passing through the sensing element. The term $g_{c}$ is a current-to-field coefficient [10].

The sensor was characterized in a magnetically shielded room, so that we may neglect $b_{\text {ext }}$. The intrinsic magnetic noise is understood to be very low [2] and is neglected in what follows. The coefficient, $g_{c}$, is evaluated by measuring the shift in impedance of the GMI wire when a DC current is applied to the detection coil. For the element described in Sec. II-A, we find $g_{c}=47 \mathrm{mT} / \mathrm{A}$. The current noise, $i_{\text {det }}$, is due to the detection stage, so that $i_{d e t}^{2}=i_{n_{b u f f e r}}^{2}$, here.

4) Sensitivity:

The sensitivity, $T_{r}$, at the sensor output expressed in $\mathrm{V} / \mathrm{T}$ is defined as

$$
T_{r}=\frac{\partial V_{\text {out }}}{\partial B}
$$

where $V_{\text {out }}$ is the voltage at the sensor output. It is easily evaluated using $\partial Z_{i j} / \partial B$ and $Z_{i j_{0}}$ of eq. 3 and eq. 10 , and 
from circuit elements and yields

$$
\begin{aligned}
T_{r} \approx & \frac{V_{g}}{2} \frac{\left|Z_{21_{0}}\left(\omega_{p}\right)\right|}{\left|Z_{11_{0}}\left(\omega_{p}\right)\right|+R_{g}}\left(\frac{\left|S_{21-\Omega}\left(\omega_{p}\right)\right|}{\left|Z_{21_{0}}\left(\omega_{p}\right)\right|}\right. \\
& \left.-\frac{\left|S_{11-\Omega}\left(\omega_{p}\right)\right|}{\left|Z_{11_{0}}\left(\omega_{p}\right)\right|+R_{g}}\right) \\
\approx & \frac{V_{g}}{2} \frac{\left|S_{21-\Omega}\left(\omega_{p}\right)\right|}{\left|Z_{11_{0}}\left(\omega_{p}\right)\right|+R_{g}}
\end{aligned}
$$

where $S_{i j-\Omega}=\partial Z_{i j} / \partial B$ is the intrinsic sensitivity as discussed in section II.

5) Equivalent magnetic noise:

Given the use of the band-pass filter at the input of the demodulator, we need consider only the fundamental of the signal. The white noise around the carrier is equivalent to narrow-band noise. Thus, the demodulation factor, $k_{e n}$, for the white noise around the carrier of a peak detector [9], [10] is

$$
k_{e n}=1 / \sqrt{2}
$$

Combining equations (11) to (22), the total noise level at the sensor output is given by

$$
\begin{aligned}
e_{n_{\text {total }}}^{2}= & k_{\text {en }}^{2}\left\{G _ { \text { buffer } } ^ { 2 } G _ { \text { filter } } ^ { 2 } \left[\left(\frac{Z_{21_{0}}\left(\omega_{p}\right)}{Z_{11_{0}}\left(\omega_{p}\right)+R_{g}}\right)^{2} e_{n g}^{2}\right.\right. \\
& \left.\left.+e_{n Q}^{2}\right]+e_{n f}^{2}\right\}+e_{n L F}^{2}+T_{r}^{2} g_{c}^{2} i_{n_{\text {Buffer }}}^{2} \\
= & 2\left\{G _ { \text { buffer } } ^ { 2 } G _ { f i l t e r } ^ { 2 } \left[( \frac { Z _ { 2 1 _ { 0 } } ( \omega _ { p } ) } { Z _ { 1 1 _ { 0 } } ( \omega _ { p } ) + R _ { g } } ) ^ { 2 } \left(e_{n 1}^{2}\right.\right.\right. \\
& \left.+e_{n R_{g}}^{2}\right)+e_{n Q}^{2}+\frac{e_{n R_{2}}^{2}}{G_{b u f f e r}^{2}}+e_{n_{b u f f}}^{2} \\
& +e_{n R_{1}}^{2}+i_{n_{b u f f}}^{2} R_{1}^{2} \\
& \left.+i_{n_{\text {buff }}}^{2}\left(Z_{22_{0}}\left(\omega_{p}\right)-\frac{Z_{12_{0}}\left(\omega_{p}\right) Z_{21_{0}}\left(\omega_{p}\right)}{Z_{11_{0}}\left(\omega_{p}\right)+R_{g}}\right)^{2}\right] \\
& \left.+e_{n_{R p o l-H F}}^{2}+G_{f i l t e r}^{2} e_{n_{R f}}^{2}\right\}+e_{n_{R d e m}}^{2} \\
& +e_{n_{R p o l-B F}}^{2}+e_{n_{A m p l}}^{2}+T_{r}^{2} g_{c}^{2} i_{n_{B u f f e r}}^{2} .
\end{aligned}
$$

We recall here that Eq. (3) gives $Z_{i j_{0}}=Z_{i j}\left(B_{0}\right)$, highlighting the dependence upon the static magnetic working point, $B_{0}$.

The equivalent magnetic noise level, $b_{n_{\text {total }}}$, of the setup, expressed in $\mathrm{T} / \sqrt{\mathrm{Hz}}$ is defined as the ratio of the electronic noise spectral density at the sensor output (in $\mathrm{V} / \sqrt{\mathrm{Hz}}$ ), to the sensitivity (in $\mathrm{V} / \mathrm{T}$ ),

$$
b_{n_{\text {total }}}=\frac{e_{n_{\text {total }}}}{T_{r} G_{b u f f e r} G_{f i l t e r}} .
$$

Using the characteristics of the impedance matrix, $[Z]$, we can now analyze the noise performance of the entire measurement chain. We use the impedance matrix parameters, $Z_{i j_{0}}$ and $S_{i j-\Omega}$, obtained from experimental measurement, as presented in ref. [9]. Nevertheless, it would be of interest to use the characteristics of the impedance matrix obtained from the model presented in Sec. II-B.

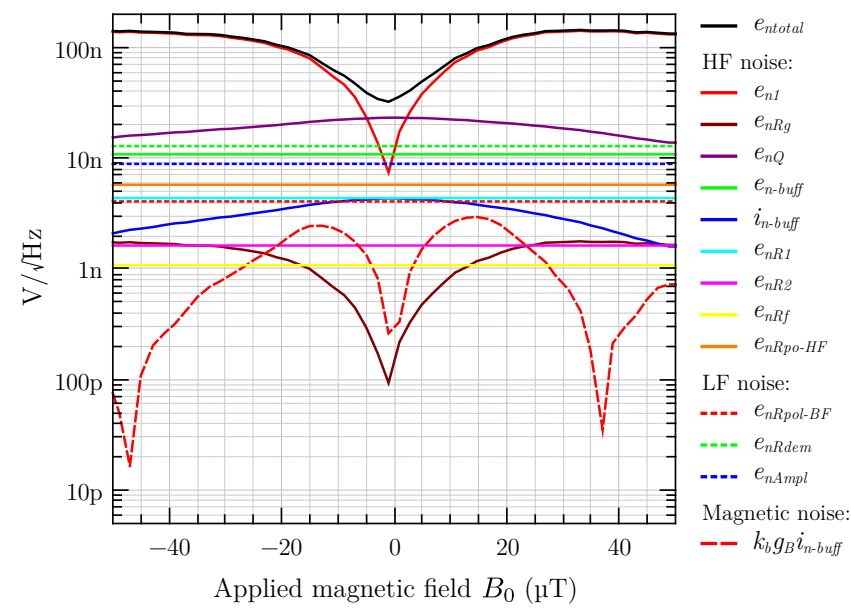

Figure 6: Details of each contribution to the total voltage noise, $e_{n_{\text {total }}}$, in the white noise region, at the output, after demodulation, as a function of the static magnetic working point, $B_{0}$. The excitation voltage amplitude, $V_{g}=10 \mathrm{Vp}$, leading to an excitation current of $13 \mathrm{~mA}$.

\section{Results}

Figure 6 presents the noise contributions of each of the sources considered in the model, as a function of the static magnetic working point, $B_{0}$. The excitation voltage amplitude, $V_{g}$, is $10 \mathrm{~V}_{\text {peak }}$, inducing an excitation current of $13 \mathrm{~mA}$ flowing through the sensing element. It may be seen that the lowest noise level is obtained by choosing an appropriate static working point near $0 \mu \mathrm{T}$. This figure helps us to analyze the dominant noise sources of the measurement chain. Here, it is dominated by the noise arising from the generator and by the Johnson noise of the resistive part of the sensing element.

The voltage sensitivity, $T_{r}$, is presented in Fig. 7 for the same excitation amplitude, $V_{g}=10 \mathrm{~V}_{\mathrm{p}}$. The maximum value, approximately $150 \mathrm{kV} / \mathrm{T}$, is obtained for a static working point, $B_{0}$, near $20 \mu \mathrm{T}$. Then, combining Figs. 6 and 7 yields the total equivalent magnetic noise, $b_{n_{\text {total }}}$, as shown in Fig. 8. It may be seen that for static working points between -20 and $+20 \mu \mathrm{T}$, noise performance on the order of $500 \mathrm{fT} / \sqrt{\mathrm{Hz}}$ in the white noise range should be achievable. Note that the asymmetric response with respect to field in Figs. 6 to 8 is due to the use of a DC bias current.

Figure 9 shows the measured noise spectral density of the measurement chain shown in Fig. 5, for several excitation amplitudes. They are expressed both as total voltage noise at the chain output, $e_{n_{\text {total }}}$ in $\mathrm{V} / \sqrt{\mathrm{Hz}}$, and as equivalent magnetic noise, $b_{n_{\text {total }}}$ in $\mathrm{T} / \sqrt{\mathrm{Hz}}$. The dashes indicate the noise due to the electronic conditioning, measured by replacing the sensing element by a resistor bridge which provides the same carrier amplitude at the buffer input, but no magnetic signal (ghost sample). In the white noise region, the noise levels, with or without sensing element, are the same. This illustrates that the white noise is dominated by electronic conditioning noise, as assumed in the model. The experimental value of sensitivity, approximately $160 \mathrm{kV} / \mathrm{T}$ for an excitation of $13 \mathrm{~mA}$, is in fair agreement with the prediction. The measured noise, $700 \mathrm{fT} / \sqrt{\mathrm{Hz}}$ in the white noise region, is 


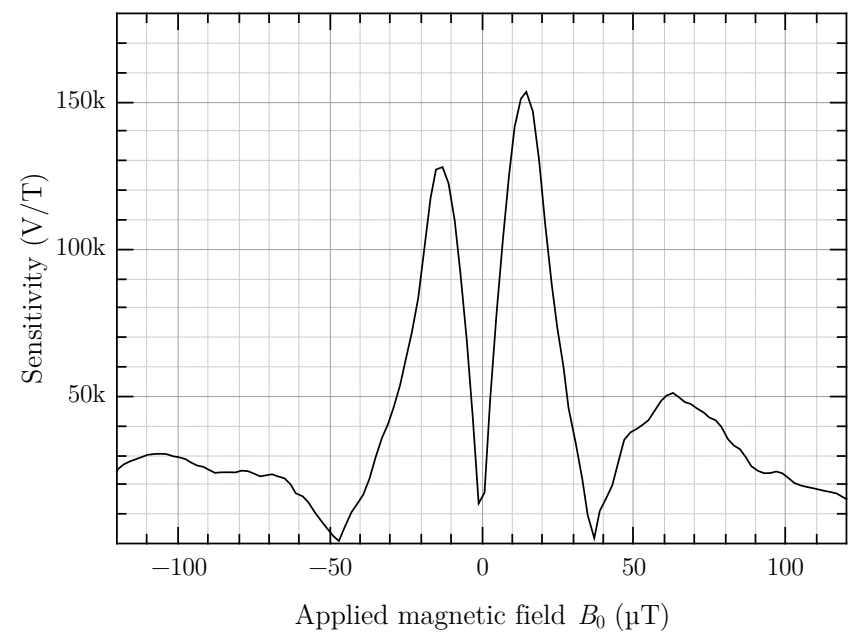

Figure 7: Sensitivity, $T_{r}$, expressed in $\mathrm{V} / \mathrm{T}$, of the measurement chain as a function of the static working point, $B_{0}$, with $V_{g}=10 \mathrm{Vp}$, which induces an excitation current of $13 \mathrm{~mA}$. The asymmetrical shape of this curve, with respect to $B_{0}=0 \mu \mathrm{T}$, is due to the asymmetrical behavior of the GMI response of the magnetic wire induced by the applied DC bias current.

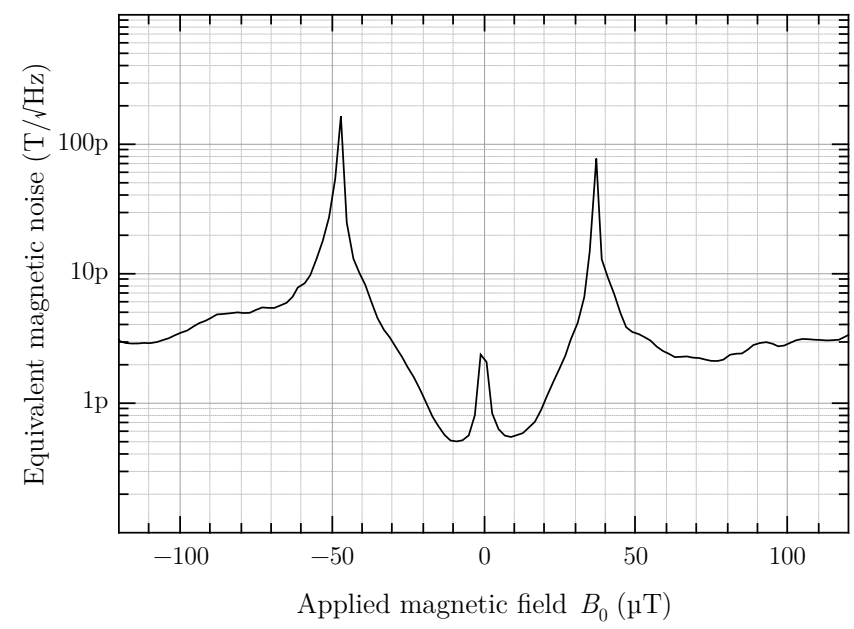

Figure 8: Equivalent magnetic noise level, $b_{n_{\text {total }}}$, in the white noise range, at the sensor output, expressed as $\mathrm{T} / \sqrt{\mathrm{Hz}}$, as a function of the static working point, $B_{0}$, with $V_{g}=10 \mathrm{~V}_{\text {peak }}$, which induces an excitation current of $13 \mathrm{~mA}$.

somewhat higher than the value of $500 \mathrm{fT} / \sqrt{\mathrm{Hz}}$ predicted from the model.

At frequencies below $1 \mathrm{kHz}$, considerable $1 / f$ noise is observed. Since the carrier level of the signal at the buffer input is the same in the three measurements, the voltage noise of the excitation generator should be the same, so that equivalent magnetic noise should be sensitivity dependent, as it is in the white noise range. Nevertheless, experimental results in the $1 / f$ range appears to be independent of sensitivity. Furthermore, it exceeds the noise from electronic conditioning, measured with the ghost sample. That is, the additional noise acts as if it is magnetic in origin. Since the magnetic noise due to $g_{c} i_{n_{b u f f}}$ is too small to explain this difference, we suggest

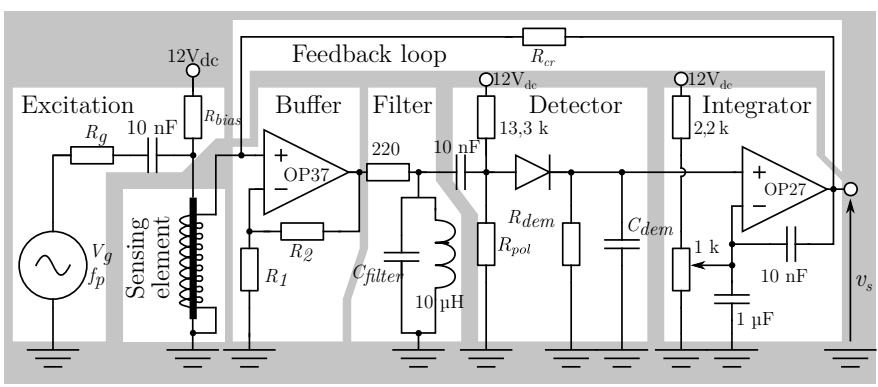

Figure 10: Schematic of the magnetometer.

Component values are: $R_{g}=750 \Omega, R_{\text {bias }}=2,2 \mathrm{k} \Omega$ (leading to $\left.I_{d c}=6 \mathrm{~mA}\right), R_{1}=100 \Omega, R_{2}=500 \Omega, C_{\text {filter }}=2,2 \mathrm{nF}$, $R_{\text {pol }}=1 \mathrm{k} \Omega, R_{\text {dem }}=10 \mathrm{k} \Omega$ et $C_{\text {dem }}=100 \mathrm{nF}$. Resistor $R_{c r}$ is adjusted depending upon the feedback coil used, in order to set the desired feedback gain, $T_{c r}$. The excitation current in the sensing element is $13 \mathrm{~mA}$ (that is $V_{g}=10 \mathrm{~V}_{\text {peak }}$ ) at $f_{p}=1 \mathrm{MHz}$.

that it arises from intrinsic GMI noise, which is neglected in our model. Cross spectrum coherence measurements, obtained using two identical measurement chains which split either upstream or downstream from the sensing element, confirm this suggestion [17]. Nevertheless, the precise origin of this $1 / f$ noise is not totally clear at this time.

\section{MAgnetometer}

As the field response of the GMI element is highly nonlinear, it must be implemented in a field feedback loop in order to obtain a true, linear, magnetometer.

\section{A. Feedback loop}

Now, the optimized measuring chain described in Sec. III-A is integrated with a field feedback loop. The corresponding schematic is shown in Fig. 10. The direct chain is followed by an integrator block, which insures that there is no static error in the output signal. This block is based on a low-noise OpAmp OP27 in pseudo-integrator configuration. An offset adjustment on the non-inverting input of this stage permits compensation of the static component at the demodulator output, related to the choice of static magnetic working point. In an equivalent way, this offset adjustment can be used to set the static working point.

The output voltage from the integrator is applied to a resistor, $R_{c r}$, in series with a coil, converting voltage to current, and induces a magnetic field proportional to this current. The sensing element is subjected to the sum of the external field to be measured and the opposing feedback field. In practice, it is possible to use two kinds of feedback coil. An external coil surrounding the entire sensing element will not affect the direct chain in any way. Alternatively, the low frequency feedback current may be applied to the detection coil, as illustrated in Fig. 10. This arrangement is more compact, and is retained here as the preferred solution. However, this introduces an impedance in parallel to the detection coil, equal to the output impedance of the $R_{c r}$-integrator assembly. This modifies the direct chain, and may influence the performance. The resistor 

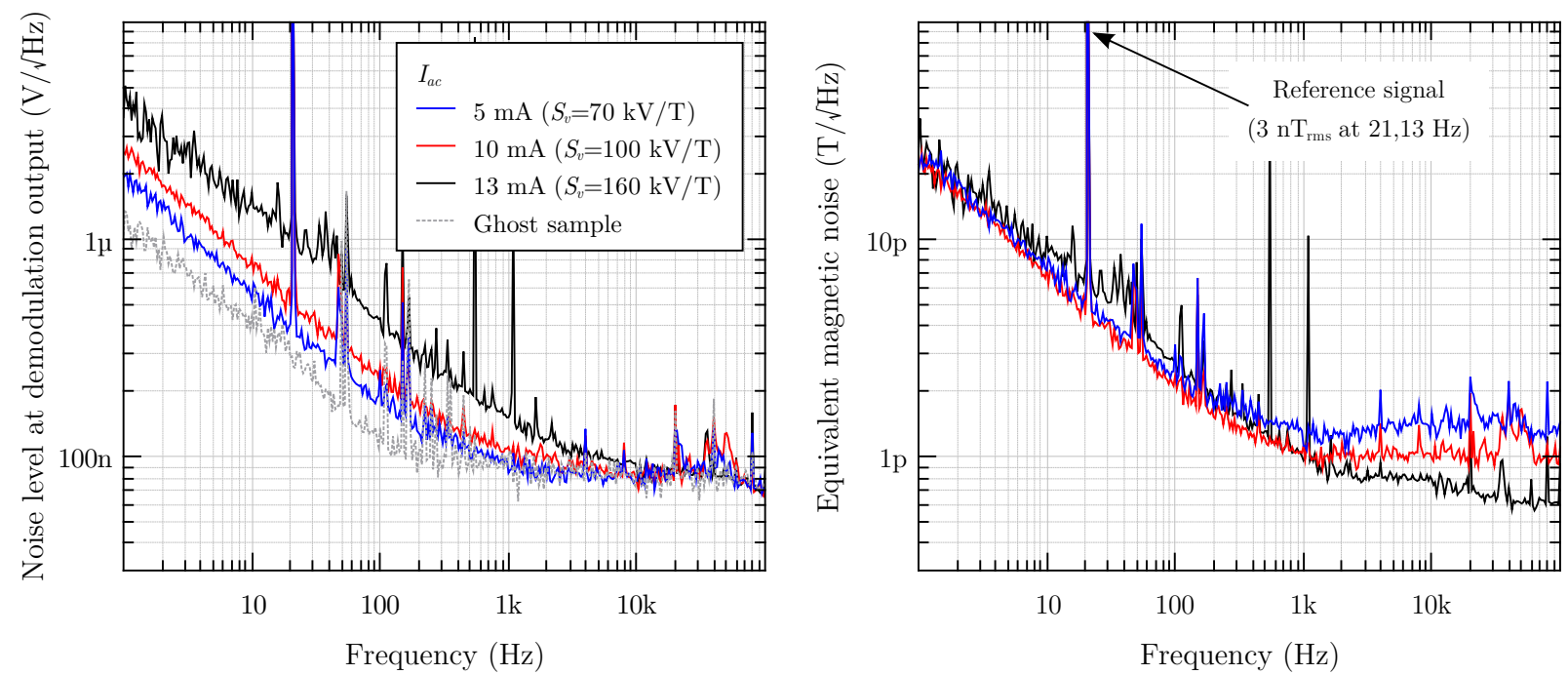

Figure 9: Voltage noise spectral densities at the output (left) and equivalent magnetic noise spectral densities (right), measured for excitation amplitudes of $V_{g}=10,7.5$ and $3.75 \mathrm{~V}_{\text {peak }}$ (corresponding to excitation currents $I_{a c}=13,10$ and $5 \mathrm{~mA}$ ). The pronounced spectral line at $20 \mathrm{~Hz}$ corresponds to the response to a reference magnetic signal. The amplitude of this spectral line at the output permits evaluation of the sensitivity in $\mathrm{V} / \mathrm{T}$.

$R_{c r}$ is adjusted to set the feedback coil transfer, which sets the feedback gain. It is the gain of the feedback loop which finally sets the sensitivity of the magnetometer (assuming that direct gain is much higher than the inverse of feedback gain).

\section{B. Performance}

An optimized magnetometer using the detection coil as feedback coil has been constructed and characterized. Here, we present its performance.

1) Noise and bandpass:

Figure 11 presents the transfer function of the off-diagonal GMI magnetometers. The feedback magnetic field is generated directly by the detection coil. The feedback resistor, $R_{c r}$, is set to $3.6 \mathrm{k} \Omega$. The current-to-field transfer, at low frequency, of this coil, is unknown a priori. The measured sensitivity of the obtained magnetometer is $100 \mathrm{kV} / \mathrm{T}$, as shown in Fig. 11. This allows us to deduce the transfer of the coil, from $\frac{R_{c r}}{100 \mathrm{kV} / \mathrm{T}}=36 \mathrm{mT} / \mathrm{A}$. Comparing this value with the estimate, $g_{c}=48 \mathrm{mT} / \mathrm{A}$, from Sec. III-B3, we conclude that they are in rough agreement. The $-3 \mathrm{~dB}$ band-pass is approximately $70 \mathrm{kHz}$.

The equivalent magnetic noise density is shown in Fig. 12. The white noise performance is degraded compared to that obtained with the open loop. This is due to the modification of the direct chain, introduced by the connection of the feedback loop to the sensor output. Indeed, the output impedance of the $R_{c r}$-integrator block degrades the input impedance of the buffer, as seen by the detection coil. Thus, the signal entering the buffer is reduced slightly, as is the sensitivity.

2) Maximum excursion and dynamics:

The maximum amplitude of measurable magnetic field is limited by the maximum output voltage of the integrator output stage. For the design presented here, this is $\pm 100 \mu \mathrm{T}$, large enough to permit compensation of the static component of geomagnetic field, allowing the use of this magnetometer

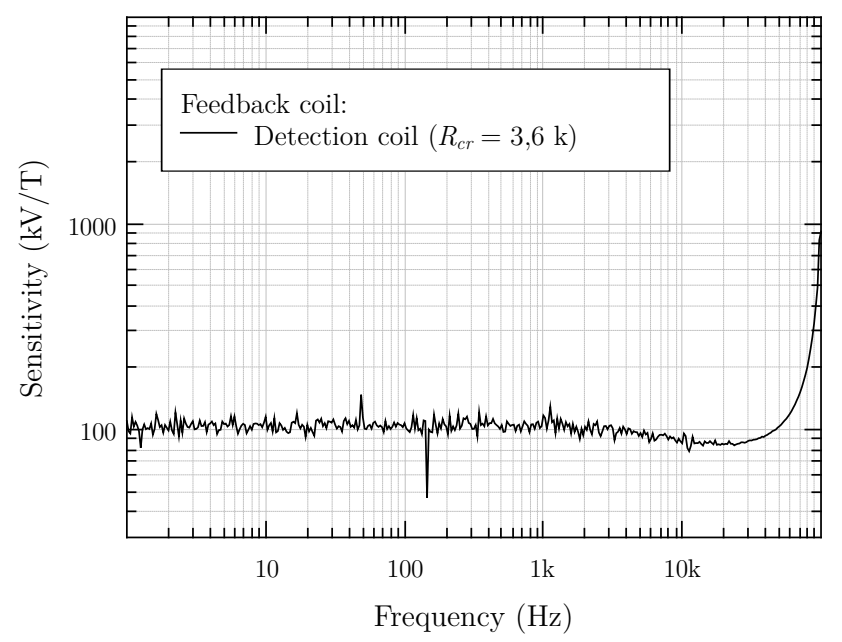

Figure 11: Transfer function of the magnetometer. We notice that the overshoot is induced by the resonance of the feedback loop.

in a non-shielded environment. Comparing this value to the equivalent magnetic noise level, it defines a dynamic range of $140 \mathrm{~dB} / \sqrt{\mathrm{Hz}}$ at $10 \mathrm{~Hz}$.

\section{CONClusion}

Following our previous finding that the GMI-coil configuration constitutes a promising approach to the improvement of noise performance of GMI based magnetometers [9], we have constructed a practical optimized magnetometer. Its performance is summarized in Table I. This optimization is based on a systematic study of the complete system, from physical properties of the GMI wire to magnetometer noise performance. This is illustrated here by the optimization of 
Table I: Measured performances of the off-diagonal GMI-based magnetometer with combined detection and feedback coil.

\begin{tabular}{lccccc}
\hline Sensitivity & Bandpass & $\begin{array}{c}\text { Noise level } \\
\text { at } 1 \mathrm{~Hz}\end{array}$ & $\begin{array}{c}\text { White noise } \\
\text { level }\end{array}$ & $\begin{array}{c}\text { Maximum } \\
\text { excursion }\end{array}$ & $\begin{array}{c}\text { Sensing element } \\
\text { dimensions }\end{array}$ \\
\hline $\mathrm{kV} / \mathrm{T}$ & $\mathrm{Hz}$ & $\mathrm{pT} / \sqrt{\mathrm{Hz}}$ & $\mathrm{pT} / \sqrt{\mathrm{Hz}}$ & $\mu \mathrm{T}$ & $\mathrm{mT} / \mathrm{s}$ \\
\hline \hline 100 & $\mathrm{DC}$ to $70 \mathrm{kHz}$ & 35 & 1.7 & \pm 100 & $\mathrm{~mm}$ \\
\hline
\end{tabular}

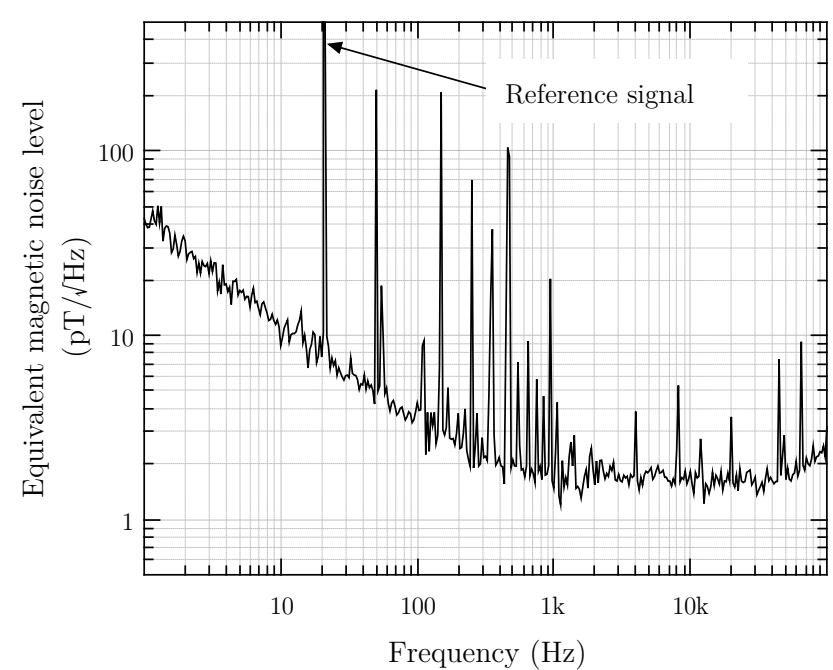

Figure 12: Equivalent magnetic noise spectral density of the magnetometer.

the detection coil design and by the model of noise. The experimental results clearly validate the theoretical approach.

Possible further advances include improvement of the excitation stage, which is presently the limiting factor in noise performance in the white noise range, improved understanding of the nature of the $1 / f$ noise at low frequency, and reduction of the parasitic capacitance of the detection coil. Nevertheless, the present design shows noise slightly above $1 \mathrm{pT} / \sqrt{\mathrm{Hz}}$ in the white noise range. This makes GMI magnetometers competitive with technologies which are widely used at present, such as the classical flux-gate magnetometers [18].

\section{REFERENCES}

[1] M. Knobel and K. Pirota, "Giant magnetoimpedance: concepts and recent progress," Journal of Magnetism and Magnetic Materials, vol. 242, pp. 33-40, 2002.

[2] L. G. C. Melo, D. Ménard, A. Yelon, L. Ding, S. Saez, and C. Dolabdjian, "Optimization of the magnetic noise and sensitivity of giant magnetoimpedance sensors," Journal of Applied Physics, vol. 103, no. 3, p. 033903, 2008.

[3] L. Ding, S. Saez, and C. Dolabdjian, "Low Frequency Giant Magnetoimpedance Magnetometer Noise Versus Electronic Conditioning," Sensor Letters, vol. 5, no. 1, pp. 248-251, 2007.

[4] L. Ding, S. Nabily, S. Saez, J. Gieraltowski, and C. Dolabdjian, "Investigation of Giant Magnetoimpedance Magnetic Noise Comparison," Sensor Letters, vol. 5, no. 1, pp. 171-175, 2007.

[5] D. Ménard, D. Seddaoui, L. G. C. Melo, A. Yelon, B. Dufay, S. Saez, and C. Dolabdjian, "Perspectives in Giant Magnetoimpedance Magnetometry," Sensor Letters, vol. 7, no. 3, pp. 339-342, 2009.

[6] B. Dufay, S. Saez, C. Dolabdjian, A. Yelon, and D. Ménard, "Physical properties and giant magnetoimpedance sensitivity of rapidly solidified magnetic microwires," Journal of Magnetism and Magnetic Materials, vol. 324 , no. 13, pp. 2091-2099, 2012.
[7] S. Sandacci, D. Makhnovskiy, L. Panina, K. Mohri, and Y. Honkura, "Off-diagonal impedance in amorphous wires and its application to linear magnetic sensors," Magnetics, IEEE Transactions on, vol. 40, no. 6 , pp. 3505-3511, 2004.

[8] E. Paperno, "Suppression of magnetic noise in the fundamental-mode orthogonal fluxgate," Sensors and Actuators A: Physical, vol. 116, no. 3, pp. 405-409, 2004.

[9] B. Dufay, S. Saez, C. Dolabdjian, A. Yelon, and D. Ménard, "Impact of electronic conditioning on the noise performance of a two-port network giant magnetoimpedance magnetometer," IEEE Sensors Journal, vol. 11, no. 6, pp. 1317-1324, 2011.

[10] L. Ding, S. Saez, C. Dolabdjian, L. G. C. Melo, A. Yelon, and D. Ménard, "Equivalent magnetic noise limit of low-cost GMI magnetometer," IEEE Sensors Journal, vol. 9, pp. 159-168, 2009.

[11] L. Landau and E. Lifshitz, Electrodynamics of continuous media. Pergamon Press, Oxford, 1960.

[12] D. Ménard and A. Yelon, "Theory of longitudinal magnetoimpedance in wires," Journal of Applied Physics, vol. 88, p. 379, 2000.

[13] D. Makhnovskiy, L. Panina, and D. Mapps, "Measurement of fielddependent surface impedance tensor in amorphous wires with circumferential anisotropy," Journal of Applied Physics, vol. 87, p. 4804, 2000.

[14] Function / Arbitrary waveform generator HP 33120A User's Guide, Hewlett-Packard Co., 1994.

[15] OP37, Low Noise, Precision, High Speed, Operational Amplifier Datasheet, Analog Devices, 2002, rev. B.

[16] INA128, Precision, Low Power, Instrumentation Amplifiers, Texas Instruments, 2005, rev. B.

[17] "GREYC internal report," Tech. Rep., 2011.

[18] B. Dufay, S. Saez, C. Dolabdjian, A. Yelon, and D. Ménard, "Development of a high sensitivity Giant Magneto-Impedance magnetometer: comparison with a commercial Flux-Gate," Submitted for publication to the proceeding of the European Magnetic Sensors and Actuators Conference, 2012. 